\title{
Role of Periodontist in Pediatric Dental Patients
}

\author{
${ }^{1}$ Harpreet Singh Grover, ${ }^{2}$ Yogender Singh, ${ }^{3}$ Amit Bhardwaj
}

\begin{abstract}
Modern age dentistry is very much different from what it was in the past. In this period of super specialization, clinical skills are tuned finely with professional expertise is improved. Interdisciplinary approach is mandatory to deal with any clinical situation to provide optimal and timely treatment results. This paper gives the emphasis on comprehensive management of young people with gingival and periodontal diseases. The paper provides the background to the condition, the possible etiological factors, the prevalence of peridontal diseases and other related conditions. Lastly, there is consideration of the role of the periodontist in interdisciplinary management of the affected child and young patient.
\end{abstract}

Keywords: Children, Gingival diseases, Periodontitis, Treatment.

How to cite this article: Grover HS, Singh $Y$, Bhardwaj A. Role of Periodontist in Pediatric Dental Patients. World J Dent 2015;6(1):49-54.

Source of support: Nil

Conflict of interest: None

\section{INTRODUCTION}

Periodontists have a significant role to play in the early detection and diagnosis of gingival and periodontal diseases in children. Both primary dental care or by reference to a periodontist will ensure the success of the treatment. Periodontal management is need of the hour for effective oral hygiene practices in childhood, adolescence and early adulthood and beyond.

Guidelines which can be followed are as follows:

- To chart a screening method for children and adolescents for periodontal examination at the initial stages.

- To decide when it is right time to treat in practice or refer to periodontist, thus, optimizing periodontal treatment outcomes for children and young adolescents.

\section{Important Features of a Healthy Periodontium}

Children having healthy gingival and periodontal status have gingival margin is few millimeters coronal to the

\footnotetext{
${ }^{1}$ Professor and Head, ${ }^{2}$ Postgraduate Student, ${ }^{3}$ Reader

${ }^{1-3}$ Department of Periodontology, Faculty of Dental Sciences SGT University, Gurgaon, Haryana, India
}

Corresponding Author: Amit Bhardwaj, Reader, Department of Periodontology, Faculty of Dental Sciences, SGT University Gurgaon, Haryana, India, Phone: 09818718872, e-mail: amitmds1980@rediffmail.com cementoenamel junction. The gingival sulcus depth ranging from 0.5 to $3 \mathrm{~mm}$ could be seen on a fully erupted tooth. In youngsters with a healthy periodontium, the alveolar bone crest is situated 0.4 to $1.9 \mathrm{~mm}$ apical to the CEJ. ${ }^{1}$

Periodontal diseases in children can be divided into two categories on the basis of their etiology.

\section{Gingival Diseases}

- Nonplaque-induced gingival disease

- Plaque-induced gingival disease

\section{Periodontitis}

- Chronic periodontitis

- Aggressive periodontitis

- Periodontitis as a manifestation of systemic diseases

\section{Gingival Diseases}

\section{Nonplaque-induced Gingival Lesions}

The nonplaque-induced gingival lesions which may be found in children are described in Table 1.

\section{Plaque-induced Gingivitis}

As a result of plaque accumulation, there is buildup of inflammatory cell infiltrate in connective of gingiva. This will result in disruption of junctional epithelium and more apical plaque deposition which in turn leads to gingival pocket formation. In severe inflammatory conditions, gingival swelling occurs and leads to increased false pocket depth. Up to this stage, apical extent of the junctional epithelium is lies at the cementoenamel junction with no loss of periodontal attachment levels. With effective plaque control, this inflammatory process is completely reversible. As dental plaque is the crucial etiological agent in development of periodontal disease, different local and systemic risk factors can modify the individual's response to plaque accumulation and manipulate the development and progression of gingival diseases to advanced stages.

Plaque-induced gingivitis can happen at any age but generally low prevalence of gingivitis is seen during preschool age with gradual increase that reaches to a peak around puberty, possibly due to changes in the inflammatory cell response, bacterial composition of the dental plaque and hormonal levels. ${ }^{2}$ 


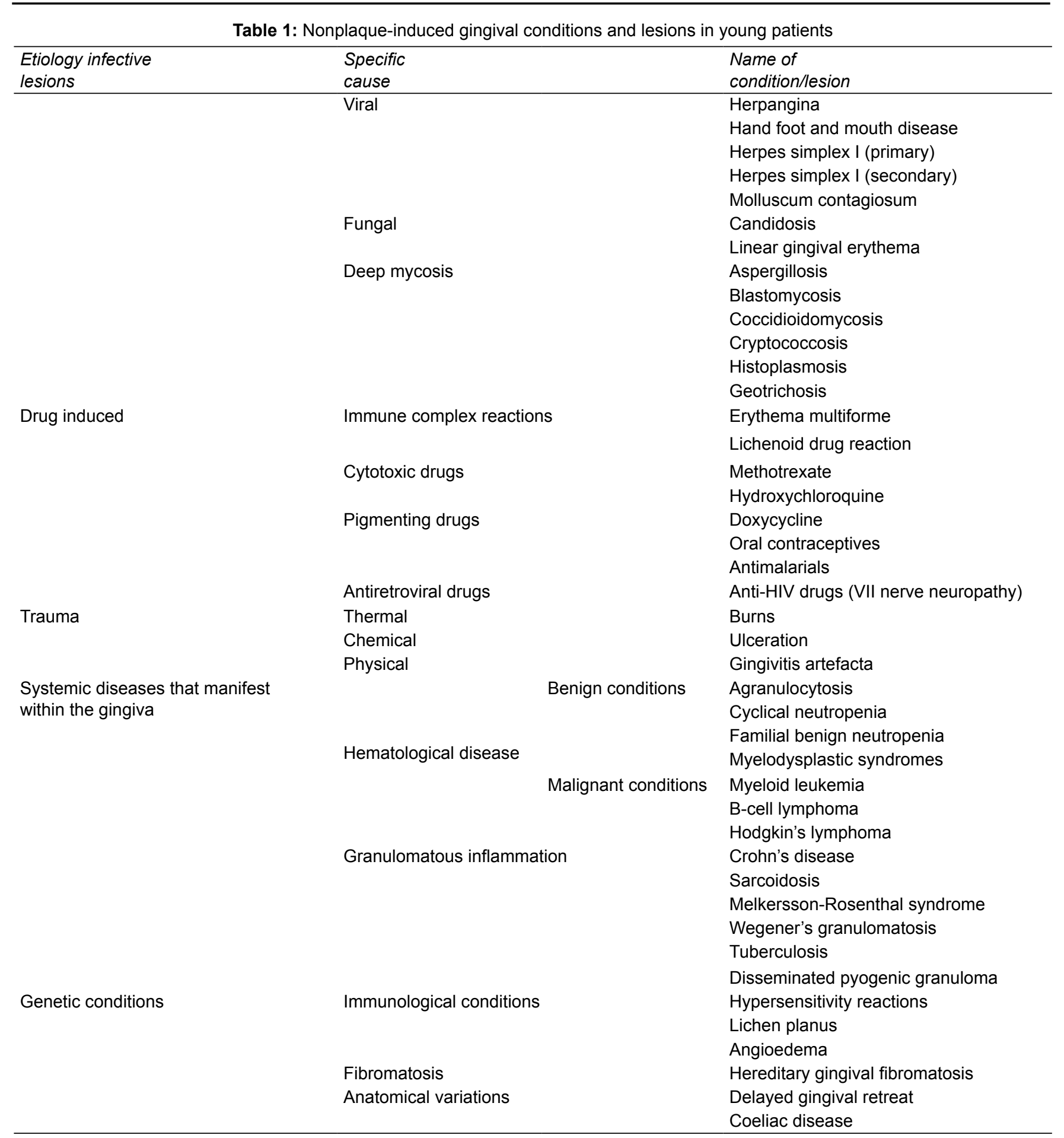

\section{Periodontitis}

The key features of periodontitis are as follows:

- Progressive destruction of periodontal ligament and alveolar bone.

- Increased probing depth formation, recession or both.

\section{Chronic Periodontitis}

A significant number of adolescents manifest attachment loss of $1 \mathrm{~mm}$ or more, consistently in initial stages of chronic periodontitis. ${ }^{3}$ Periodontopathogens present in the subgingival microflora of teenagers with incipient chronic periodontitis are namely Porphyromonas gingivalis, Aggregatibacter actinomycetemcomitans and Prevotella intermedia. ${ }^{4}$ A 3 years longitudinal study in adolescents shows that Tannerella forsythia has been associated with clinical attachment loss. ${ }^{5}$

\section{Aggressive Periodontitis}

Mostly affects systemically healthy individuals aged less than 30 years. According to consensus report of the 1999 International Workshop, following common and secondary features is present in aggressive periodontitis. ${ }^{6}$ 


\section{Common Features}

- Patients are clinically healthy other than periodontitis.

- Bone destruction with rapid loss of attachment levels.

- Familial aggregation of diseased individuals.

\section{Secondary Features}

- Quantity of microbial deposits is not consistent with the severity of periodontal destruction.

- Elevated proportions of A. actinomycetemcomitans and the levels of $P$. gingivalis may also be affected.

- Abnormalities in function of phagocytes.

- Hyper-responsive macrophages, producing increased prostaglandin $\mathrm{E}_{2}$ and interleukin- $1 \beta$.

- Progression of attachment and bone loss may be self limiting.

Aggressive peridontitis may be further classified into localized and generalized form with following specific features:

Localized form

- Circumpubertal onset

- Robust serum antibody response to infecting agent.

- Localized first molar or incisor disease with proximal attachment loss on at least two permanent teeth, one of which is a first molar.

Generalized form

- Frequently seen in people aged less than 30 years but they may be older, infrequently occurs in teenagers

- Poor serum antibody response is infecting agents

- Pronounced episodic nature of the periodontal destruction

- Generalized interproximal loss of attachment affecting at least three teeth excluding first molars and incisors.

Periodontologist or pediatric dentist should be consulted for patients with aggressive periodontitis (Table 2).

Table 2: Referring to a periodontist

When to refer to a specialist?

- Diagnosis of aggressive periodontitis

- Incipient chronic periodontitis not responding to treatment

- Systemic medical condition associated with periodontal destruction

- Medical history that significantly affects periodontal treatment or requiring multidisciplinary care

- Genetic conditions predisposing to periodontal destruction

- Root morphology adversely affecting prognosis

- Nonplaque-induced conditions requiring complex or specialist care

- Cases requiring diagnosis/management of rare/complex clinical pathology

- Drug-induced gingival overgrowth

- Cases requiring evaluation for periodontal surgery

\section{Periodontal Screening during Different Types of Dentitions}

\section{Primary Dentition}

Evidence from various retrospective epidemiological study data shows the presence of bone loss around the primary dentition radiographically, which reinforce the concept that periodontitis can occur even at early age. ${ }^{7}$

\section{Mixed Dentition}

During mixed dentition period, it is of prime importance for the practitioner to be well aware of false pocketing in partially erupted teeth.

\section{Permanent Dentition}

In young adults, puberty gingivitis is mostly seen which results mainly due to the increased inflammatory response of gingival to dental plaque modified by the hormonal changes related with puberty. The shift from gingival disease to the early stages of periodontitis may occur in early teenage years. Which may be characterized by loss of 1 to $2 \mathrm{~mm}$ clinical attachment interproximally, 4 to $5 \mathrm{~mm}$ deep periodontal pockets and loss of crestal alveolar bone about $0.5 \mathrm{~mm}$ which is mostly horizontal. Different factors either local or systemic may influence rate, severity and extent of progression of periodontal diseases (Flow Chart 1).

\section{Basic Periodontal Examination in the Primary Dental Care ${ }^{8}$}

All new patients aged less than 18 years and those undertaking orthodontic treatment in the mixed or permanent dentition with full eruption of index teeth (all four first permanent molars plus upper right permanent central incisor, lower left permanent central incisor) should have

Flow Chart 1: Periodontal screening chart

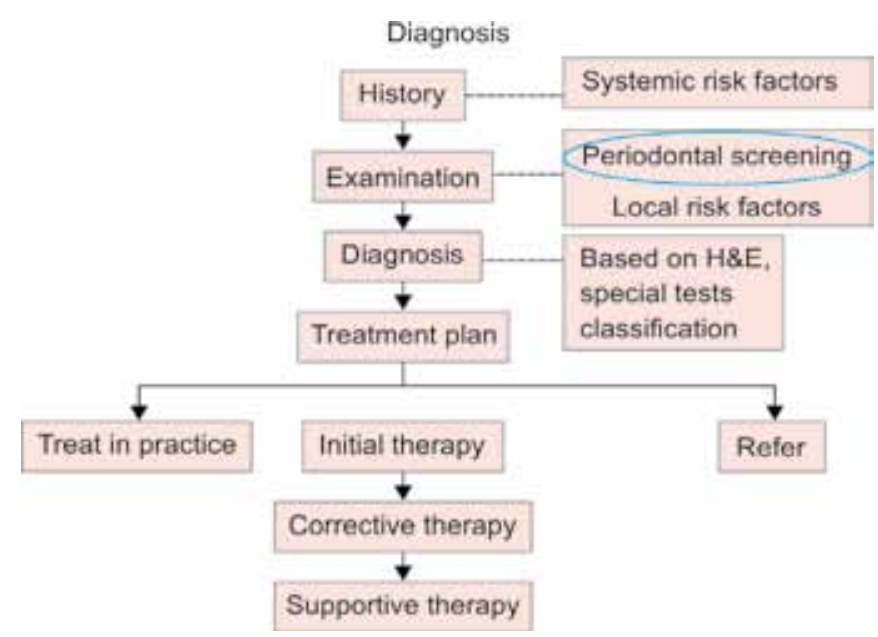


Table 3: Basic periodontal examination

code and management options

\begin{tabular}{ll}
\hline BPE code & Management options \\
\hline 0 & $\begin{array}{l}\text { Appropriate preventive care } \\
\text { Chart gingival bleeding. Disclose and } \\
\text { chart plaque. Oral hygiene instructions. }\end{array}$ \\
& $\begin{array}{l}\text { Prophylaxis } \\
\text { Chart gingival bleeding. Disclose and chart } \\
\text { plaque. Oral hygiene instructions. Remove } \\
\text { defective margins, plaque retention factors. }\end{array}$ \\
& $\begin{array}{l}\text { Scale and prophylaxis } \\
3\end{array}$
\end{tabular}

Manage as for code 2, plus record probing depths and bleeding on probing on affecting index tooth (6 sites)-should also check if any other teeth in sextent are affected. Treatment will take longer and include scale and root surface debridement (RSD). Consider referral if poor response Full periodontal charts. Oral hygiene instructions. Remove defective margins, plaque retention factors. Scale and RSD as appropriate. Consider referral to specialist

*With 0,1, 2 As for code 0, 1, 2 above, plus periodontal charts of furcation and treat as appropriate. Consider referral to specialist

*With 3, $4 \quad$ Full periodontal charts. Scale, prophylaxis and RSD as appropriate. Consider specialist referral

${ }^{*}$ Presence of furcation defect along with other numbers

the simplified basic periodontal examination recorded, where this is deemed to be appropriate, taking into account patient cooperation and level of anxiety. The following guide is intended to aid patient management (Table 3).

\section{Management of Index Teeth according to Simplified BPE Code}

- Code 0: No treatment required.

- If $\mathrm{BPE}=0$, screen again at routine recall visit or within 1 year, whichever the sooner.

- Code 1: Oral hygiene instruction and prophylaxis

- Code 2: Supra and subgingival scaling at selected sites in addition to oral hygiene instruction and prophylaxis. Remove plaque retention factors.

- If $\mathrm{BPE}=1$ or 2 , treat and screen again at routine recall or after 6 months, whichever the sooner.

- Code 3,4 and * following full periodontal assessment, supplementary radiographs may be required to assist diagnosis, although the existence of false pocketing in the case of erupting teeth in the mixed and early permanent dentition must be considered as the gingival margin may be situated coronal to the cementoenamel junction by a number of millimeters in young individuals. Other clinical signs of pathology, e.g. bleeding, suppuration, tooth mobility, will be pertinent to an accurate diagnosis.
- After false pocketing is accounted for, young patients scoring code 3 should be treated as for code 2 except that more intensive treatment (including root surface debridement) may be indicated followed by a review after 3 months.

- Codes 4 and * are unusual in young patients and full periodontal assessment with a referral to a Specialist, Periodontologist or Pediatric Dentist should be considered.

\section{Oral Healthcare Measures}

\section{Motivation}

It has been shown that professional support to patients and parents in the form of preventive/educational programs improves patient motivation, leading to improved levels of oral health. ${ }^{9}$

A review of the literature has suggested that oral health education programs may reduce plaque and gingival bleeding in the short-term only; ${ }^{10}$ however, dentists have an ethical imperative to advice patients with regards to improving oral health.

\section{Toothbrushing}

Plaque-induced chronic gingivitis in children and adolescents can be managed by mechanical removal of plaque and good oral hygiene which, additionally, has further benefits in terms of reduction of caries risk. These recommend that toothbrushing commences as soon as the first primary tooth erupts. Children less than 3 years of age should use toothpaste containing no less than $1000 \mathrm{ppm}$ fluoride, whilst family toothpaste (1350-1500 ppm fluoride) is indicated for maximum caries control in patients above 3 years of age, with adequate parental supervision as the use of small amounts are stipulated has been shown to be better than any other, rather the need to systematically clean all tooth surfaces should be emphasized by the clinician. The patient's existing toothbrushing technique may need to be modified to clean all tooth surfaces as no other toothbrushing technique has been found to be better than other. It is recognized that disclosing tablets indicate areas that are being missed. It is recommended that toothbrushing is carried out twice a day with fluoridated toothpaste.

\section{Toothbrush Type}

In adults, it has been shown that systematic, twice daily manual toothbrushing is most effective with a smallheaded toothbrush which has soft round-ended filaments compactly arranged at an angle of long and short filaments and a comfortable handle. ${ }^{11}$ An appropriate sized toothbrush should be recommended for children and 
adolescents. The periodontist can, thus, recommend good effective brushing with a manual or powered toothbrush twice daily using fluoridated toothpaste. The choice of toothbrush may be influenced by patient preference.

\section{Fixed Orthodontic Appliances}

It is essential to assess the periodontal condition of the young person before undertaking orthodontic treatment. High plaque accumulation has been described in patients undergoing therapy with fixed orthodontics. It is well recognized that plaque in association with fixed appliances can result in clinical problems, such as demineralization of the adjacent enamel and gingival inflammation. Indeed, it has been proposed that the clinical attachment level (sum of gingival recession and probing pocket depth) is a good parameter for the objective and long-term evaluation of oral health status, as it has been shown to have a close correlation with white spot lesion status. ${ }^{12}$ It is recommended that patients accepted for orthodontic treatment demonstrate an adequate level of oral hygiene, particularly in the case of those patients requiring fixed appliance therapy. Professional support and education of patients in oral hygiene practices is paramount. Toothbrushing using the Bass technique with supplementary use of approximal brushes is recommended. The orthodontic specialist is responsible for monitoring the health of both teeth and periodontal structures during the course of treatment and can use treatment visits to re-emphasize the importance of good oral hygiene practices throughout the duration of fixed appliance therapy.

\section{Flossing}

Whilst evidence relating to the effectiveness of flossing in children for the improvement in gingival and periodontal health is sparse, a comprehensive literature review has shown that regular flossing of children's teeth by a trained adult can dramatically reduce interproximal caries in those at high risk of caries. ${ }^{13}$

As for toothbrushing, with fluoridated toothpaste, there is no doubt that the benefits of interdental flossing include a reduction in the caries experience of children and adolescents. It may be beneficial to recommend supervised flossing of children's teeth for those at high risk of caries.

\section{Mouthrinses}

Mouthwashes have been shown to improve oral hygiene status and gingival health; ${ }^{14}$ however, their use is not recommended in young children who are unable to spit effectively. In addition, ethanol-containing products cannot be recommended for use in children on a longterm basis as a result of long-term safety concerns, e.g. carcinogenesis. Implementation of good toothbrushing supported by professional prophylaxis and scaling is the mainstay for the maintenance of good gingival and periodontal health.

\section{Gingival Overgrowth}

Gingival overgrowth can be related to systemic and metabolic diseases, genetic factors, local factors and side effects produced by some medications (cyclosporin, phenytoin and calcium channel blockers).

A greater incidence of gingival overgrowth is seen in puberty and the severity is more intense in children than in adults with similar amounts of dental plaque. ${ }^{15}$

Treatment for gingival overgrowth should begin with rigorous homecare and frequent appointments for scaling and professional plaque removal. Although this often leads to improvement, surgery may be necessary to correct the gingival contour, especially with respect to drug-induced gingival overgrowth; the management requires referral to periodontal specialists.

\section{Mucogingival Problems}

During eruption of the permanent tooth, there is an increase in the width of the attached gingiva. ${ }^{16}$ Findings from the literature do suggest that mucogingival surgery is not needed before the patient reaches adulthood. ${ }^{17}$ Referral to a specialist in periodontology should be considered.

\section{CONCLUSION}

Early detection of periodontal diseases in the child and adolescent population is of paramount importance for accurate diagnosis of dental, periodontal or possible underlying medical pathology and for the optimum outcome of treatment provided. The routine use of the simplified BPE on index teeth (first permanent molars, UR1 and LL1) for all cooperative child and adolescent patients under 18 years of age should form the basis of a suitable periodontal screening examination for use in the primary dental care setting when attending for the first time, at recall or prior to orthodontic therapy. In the case of the mixed and young permanent dentition, false pocketing in a dynamically erupting dentition may make accurate diagnosis of periodontal problems challenging. This should be minimized by using the six index teeth. It should, however, be recognized that BPE codes 4 and *

*Presence of furcation defect along with other numbers 
are unusual in children and adolescents under 18 years of age, and these codes, particularly in the presence of bleeding, suppuration and/or tooth mobility should prompt consideration for referral to a periodontist.

Identification of periodontal disease in the primary dentition is unusual and young children with unexplained premature exfoliation, gross mobility of primary teeth or red, edematous gingiva and suppuration for which no other dental cause can be seen should be referred for periodontal advice.

\section{REFERENCES}

1. Hausmann E, Allen K, Clerehugh V. What alveolar crest level on a bitewing radiograph represents bone loss? J Periodontol 1991;62(9):570-572.

2. Bimstein E, Matsson L. Growth and development considerations in the diagnosis of gingivitis and periodontitis in children. Pediatr Dent 1999;21(3):186-191.

3. Clerehugh V, Lennon MA, Worthington HV. Five-year results of a longitudinal study of early periodontis in 14 to 19-yearold adolescents. J Clin Periodontol 1990;17(10):702-708.

4. Clerehugh V, Seymour GJ, Bird PS, Cullinan M, Drucker DB, Worthington HV. The detection of actinobacillus actinomycetemcomitans, porphyromonas gingivalis and prevotella intermedia using an ELISA in an adolescent population with early periodontitis. J Clin Periodontol 1997;24(1):57-64.

5. Hamlet S, Ellwood R, Cullinan M, Worthington H, Palmer J, Bird P, Narayanan D, Davies R, Seymour G. Persistent colonisation with Tannerella forsythensis and loss of attachment in adolescents. J Dent Res 2004;83(3):232-235.

6. Matsson L, Hjersing K, Sjödin B. Periodontal conditions in Vietnamese immigrant children in Sweden. Swed Dent J 1995;19(3):73-81.
7. Lang M, Bartold PM, Cullinan M, Jeffcoat M, Mombelli A, Murakami S, Page R, Papapanou P, Tonetti M, Van Dyke T. Consensus report: aggressive periodontitis. Ann Periodontol 1999;4(1):53.

8. Clerehugh V. Periodontal diseases in children and adolescents. Br Dent J 2008;204(8):469-471.

9. Hochstetter AS, Lombardo MJ, D'eramo L, Piovano S, Bordoni N. Effectiveness of a preventive programme on the oral health of pre-school children. Promot Educ 2007;14(3):155-158.

10. Watt RG, Marinho VC. Does oral health promotion improve oral hygiene and gingival health? Periodontol 2000;2005;37(1): 35-47.

11. van der Weijden GA, Hioe KP. A systematic review of the effectiveness of self-performed mechanical plaque removal in adults with gingivitis using a manual toothbrush. J Clin Periodontol 2005;32(Suppl 6):214-228.

12. Lovrov S,HertrichK, Hirschfelder U. Enamel demineralization during fixed orthodontic treatment-incidence and correlation to various oral hygiene parameters. J Orofac Orthop 2007;68(5):353-363.

13. Hujoel PP, Cunha-Cruz J, Banting DW, Loesche WJ. Dental flossing and interproximal caries: a systematic review. J Dent Res 2006;85(4):298-305.

14. Axelsson P, Lindhe J. Efficacy of mouthrinses in inhibiting dental plaque and gingivitis in man. J Clin Periodontol 1987;14(4):205-212.

15. Tiainen L, Asikainen S, Saxen L. Puberty-associated gingivitis. Comm Dent Oral Epidemiol 1992;20(2):87-89.

16. Bimstein E, Eidelman E. Morphological changes in the attached and keratinized gingival and gingival sulcus in the mixed dentition period: a 5 years longitudinal study. J Clin Periodontol 1988;15(3):175-179.

17. Bosnak A, Jorgić-Srdjak K, Marcević T, Plancak D. The width of the clinically-defined keratinized gingival in the mixed dentition. ASDC J Dent Child 2002;69(3):266-270. 